\title{
Evaluating solar radiation forecast uncertainty
}

\author{
Minttu Tuononen ${ }^{1}$, Ewan J. O'Connor ${ }^{1,2}$, and Victoria A. Sinclair ${ }^{3}$ \\ ${ }^{1}$ Finnish Meteorological Institute, Helsinki, Finland \\ ${ }^{2}$ Department of Meteorology, University of Reading, Reading, UK \\ ${ }^{3}$ Institute for Atmospheric and Earth System Research/Physics, Faculty of Science, University of Helsinki, Helsinki, Finland
}

Correspondence: Minttu Tuononen (minttu.tuononen@vaisala.com)

Received: 1 November 2018 - Discussion started: 13 November 2018

Revised: 13 January 2019 - Accepted: 16 January 2019 - Published: 14 February 2019

\begin{abstract}
The presence of clouds and their characteristics have a strong impact on the radiative balance of the Earth and on the amount of solar radiation reaching the Earth's surface. Many applications require accurate forecasts of surface radiation on weather timescales, for example solar energy and UV radiation forecasts. Here we investigate how operational forecasts of low and mid-level clouds affect the accuracy of solar radiation forecasts. A total of 4 years of cloud and solar radiation observations from one site in Helsinki, Finland, are analysed. Cloud observations are obtained from a ceilometer and therefore we first develop algorithms to reliably detect cloud base, precipitation, and fog. These new algorithms are widely applicable for both operational use and research, such as in-cloud icing detection for the wind energy industry and for aviation. The cloud and radiation observations are compared to forecasts from the Integrated Forecast System (IFS) run operationally and developed by the European Centre for Medium-Range Weather Forecasts (ECMWF). We develop methods to evaluate the skill of the cloud and radiation forecasts. These methods can potentially be extended to hundreds of sites globally.

Over Helsinki, the measured global horizontal irradiance (GHI) is strongly influenced by its northerly location and the annual variation in cloudiness. Solar radiation forecast error is therefore larger in summer than in winter, but the relative error in the solar radiation forecast is more or less constant throughout the year. The mean overall bias in the GHI forecast is positive $\left(8 \mathrm{~W} \mathrm{~m}^{-2}\right)$. The observed and forecast distributions in cloud cover, at the spatial scales we are considering, are strongly skewed towards clear-sky and overcast situations. Cloud cover forecasts show more skill in winter when the cloud cover is predominantly overcast; in summer there are more clear-sky and broken cloud situations.
\end{abstract}

A negative bias was found in forecast GHI for correctly forecast clear-sky cases and a positive bias in correctly forecast overcast cases. Temporal averaging improved the cloud cover forecast and hence decreased the solar radiation forecast error. The positive bias seen in overcast situations occurs when the model cloud has low values of liquid water path (LWP). We attribute this bias to the model having LWP values that are too low or the model optical properties for clouds with low LWP being incorrect.

\section{Introduction}

Accurate forecasts of solar radiation are valuable for solar energy, such as predicting power generation 1 day ahead for energy markets, and for public health reasons, such as forecasting the amount of UV radiation. The amount of solar radiation at the surface is highly dependent on the solar zenith angle and clouds. However, clouds are highly variable in space and time, as are their optical properties, and therefore solar radiation forecasts require accurate cloud forecasts. Many applications only require reliable climatologies of the solar resource, such as solar resource assessments for solar energy installations (Kleissl, 2013). Observed climatologies can be obtained from surface-based instrumentation (Ohmura et al., 1998) and from satellite (Posselt et al., 2012; López and Batlles, 2014; Müller et al., 2015). Climatologies can also be derived from Numerical Weather Prediction (NWP) forecasts and reanalyses, which are attractive from a cost perspective but may display larger uncertainties than observations (Jia et al., 2013; Boilley and Wald, 2015; Frank et al., 2018; Urraca et al., 2018). Climatologies require that the correct amount and type of cloud is predicted on average, whereas a 
forecast additionally requires that the cloud is forecast at the right time.

Evaluating cloud forecasts and their impact on solar radiation has been performed using ground-based observations; Ahlgrimm and Forbes (2012) investigated the impact of low clouds on solar radiation in the Integrated Forecast System (IFS) of the European Centre for Medium-Range Weather Forecasts (ECMWF) at the Atmospheric Radiation Measurement (ARM) Southern Great Plains (SGP) site in the US using cloud radar, micropulse lidar, and surface radiation measurements; Van Weverberg et al. (2018) investigated the positive temperature bias in the lower troposphere at SGP in nine different models, which was attributed to an overestimate of the net surface shortwave radiation arising from incorrectly modelled cloud radiative effects. Earlier studies also suggest that supercooled liquid layers are not correctly represented in NWP models (Ahlgrimm and Forbes, 2012; Forbes and Ahlgrimm, 2014).

Continuous verification of the vertical representation of clouds in forecast models is available through Cloudnet (Illingworth et al., 2007); however, this requires comprehensive ground-based cloud observing systems, e.g. ARM (Mather and Voyles, 2013) and Cloudnet, which are sparsely distributed across the globe. Verification of the columnintegrated cloud amount (cloud cover) can be performed at many more locations using operational SYNOP and/or ceilometer observations (Mittermaier, 2012). Ceilometers are much more widely distributed than cloud radars as they are also present at airports to detect clouds, especially liquid layers. Operationally most ceilometers only provide cloud base height and cloud amount, but in principle all ceilometers observe the attenuated backscatter profile. This profile can be further processed to yield information on the boundary layer and the presence of aerosol, liquid, ice, and precipitation (Hogan et al., 2003; Morille et al., 2007; Münkel et al., 2007; Van Tricht et al., 2014; Kotthaus and Grimmond, 2018). Manufacturer-provided cloud base algorithms are typically not public and have been developed for aviation purposes based on decreased visibility. Cloud base height has also been derived from a microphysical point of view from the attenuated backscatter profile (e.g. Illingworth et al., 2007; Martucci et al., 2010; Van Tricht et al., 2014). Our goal is to increase the cloud information available from the ceilometer attenuated backscatter profile and combine this with surface radiation measurements.

Ceilometers are often operated in large networks (e.g. by national weather services; Illingworth et al., 2015), which are now being incorporated within harmonized pan-continental networks such as E-PROFILE (Illingworth et al., 2019), through which the profile is being recorded. Thus, implementing ceilometer methods for evaluating cloud and radiation model forecasts would be a beneficial addition to the more comprehensive but sparse cloud profiling.

Our aim is to understand how the forecast of low and midlevel clouds in an NWP model impacts the forecast of so- lar radiation at the surface. Moreover, our goal is a methodology that can be implemented rapidly at numerous sites with autonomous and robust instrumentation, i.e. combining ceilometer and solar radiation observations (Sect. 2) with single-level fields from NWP models ("single level" refers to surface fields and column-integrated fields). This requires accurate detection of liquid water clouds, precipitation, ice, and fog. In Sect. 3, we detail how we improved liquid cloud detection, and developed precipitation and fog identification algorithms, for ceilometers. In this study, we concentrated on evaluating the ECMWF IFS. Details on the model and the forecast cloud and solar radiation parameters investigated are described in Sect. 4. Since we are comparing point measurements from the ceilometer and ground-based solar radiation instruments with the single-level output from gridded model data, both observations and forecast model parameters require post-processing before model evaluation. This postprocessing methodology is presented in Sect. 5 and would be applicable to a wide range of NWP models and at hundreds of observation sites globally. We use 4 years of cloud cover and solar radiation observations from Helsinki, Finland (Sect. 6), to investigate the skill of the IFS in forecasting clouds and radiation using our methodology (Sects. 7-9), whereby we explicitly examine how the skill in forecasting cloud is related to the solar radiation forecast error.

\section{Ceilometer and solar radiation observations}

A ceilometer is an active instrument, which sends very short light pulses produced by a laser into the atmosphere and detects the backscattered signal from aerosol particles, cloud droplets, and ice crystals. In this study we use a Vaisala CL51 ceilometer for observing clouds, which has a wavelength close to $910 \mathrm{~nm}$. Operationally, the instrument reports cloud base heights and cloudiness values (oktas), but the internal algorithms do not determine cloud type, such as whether the cloud contains liquid or ice or both, and therefore we do not use these values. In addition to the standard cloud reporting, ceilometers can also provide the attenuated backscatter profile, from which it is possible to distinguish liquid layers, ice clouds, fog, and precipitation; we describe the algorithms developed for this in Sect. 3. In this study, the vertical range resolution of the ceilometer is $10 \mathrm{~m}$, with attenuated backscatter profiles output every $15 \mathrm{~s}$ and a maximum range of $15 \mathrm{~km}$. The calibration of the raw attenuated backscatter profiles is performed using the method of O'Connor et al. (2004), and the background noise is identified and removed based on the signal-to-noise ratio. The noise is calculated from the furthest range gates and assumed to be constant over the profile. The identification of high ice clouds is improved through temporal and spatial averaging to increase sensitivity; however, there are still challenges in identifying high ice clouds, especially during the day when the solar background noise is 
high. Note that we take into account the ceilometer data postprocessing methods recommended by Kotthaus et al. (2016).

The ceilometer is suited to the identification of liquid clouds and precipitation in the vertical profile; however, the measurement is usually limited to the lowest liquid cloud layer due to strong attenuation, and no information is available above this layer. Figure 1a shows an example of calibrated, background-noise-removed ceilometer attenuated backscatter profiles during $9 \mathrm{~h}$ at Helsinki, Finland, on 30 March 2016. A fog layer has been identified from 08:00 to 09:45 UTC with no information available above. Liquid cloud layers have been identified between 10:30-11:00 UTC (below $1 \mathrm{~km}$ ) and 11:00-13:30 UTC (below $2.5 \mathrm{~km}$ ), again with no information available above, except around 12:30 UTC when the liquid layer is dissipating. The signal is also attenuated in the case of heavy precipitation in which the ceilometer may not detect the cloud base above the precipitation layer. Precipitation, here in the form of ice, is visible in Fig. 1a at 10:00-10:30, 13:3016:00, and after 16:30 UTC and does not reach the ground. Weak backscatter from aerosol in the boundary layer (orange colour) is visible when there is no precipitation, fog, or liquid layers close to the ground. Since the ceilometer reliably detects the first cloud layer, we can use the data to derive robust cloud cover quantities even though we cannot say if there is any more cloud above the first layer detected.

Solar radiation, specifically global horizontal irradiance (GHI), is measured with a Kipp \& Zonen CM11 Secondary Standard pyranometer. Automated quality control has been applied by the Finnish Meteorological Institute (FMI) together with a visual check to ensure the data quality. The automated quality control is based on the Baseline Surface Radiation Network (BSRN) quality control procedure (Long and Shi, 2008) with small modifications to be more suitable for Finnish conditions (Rontu and Lindfors, 2018). GHI measurements are stored as $1 \mathrm{~min}$ averages in the FMI database.

\section{Ceilometer algorithm development}

\subsection{Liquid layer identification improvements}

In this study, we develop an algorithm to detect liquid cloud layers. The Cloudnet (Illingworth et al., 2007) approach for detecting the liquid cloud base is used as a starting point. The Cloudnet approach relies on the shape of the attenuated backscatter profiles, as it is known that the liquid droplets result in a high backscatter signal and the signal attenuates in the liquid layer (Fig. 1c). Thus, liquid layers display local peaks of stronger signal in the vertical profile of attenuated backscatter coefficient $\beta$. The Cloudnet approach searches for the lowest height range gate at which the attenuated backscatter value exceeds the given threshold $\left(\beta=2 \times 10^{-5} \mathrm{~m}^{-1} \mathrm{sr}^{-1}\right.$, representing liquid and called a pivot) and the signal is attenuated $250 \mathrm{~m}$ above the pivot value. If the signal attenuates above the pivot value, the cloud base is found below the pivot value based on the gradient in the $\beta$ profile. Multiple liquid cloud bases are allowed in the Cloudnet method. This method is part of the Cloudnet approach for identifying "droplet bits" within the categorization process (Illingworth et al., 2007) and is described in detail here: http://www.met.rdg.ac.uk/ swrhgnrj/publications/ categorization.pdf (last access: 1 November 2018), under the section "3.4.2 Droplet bit".

The Cloudnet approach is skilful in situations when there is no precipitation. During strong precipitation the attenuated backscatter coefficient may exceed the given threshold used in the Cloudnet droplet bit algorithm, even if stronger values representing the true liquid layer would be present above. Therefore, the cloud base may incorrectly be identified inside the precipitation layer below the true liquid cloud base (Fig. 2a). The liquid cloud base might not be always visible due to the attenuation of the signal in a heavy precipitation layer. We improved the method to enable reliable detection in all cases, including heavy precipitation.

The algorithm for finding liquid layers relies on the same principles as the Cloudnet approach. However, our approach for finding the strong $\beta$ value (pivot), representing the liquid layer, differs. Our updated liquid layer identification relies more on the shape of the profile than an absolute threshold value and the fact that a liquid layer exhibits a strong peak in the attenuated backscatter profile. Therefore, the maximum of a localized peak value of $\beta$ is found (not only the first value above a certain threshold) with the requirement that the magnitude of the local maximum exceeds the same threshold $\beta$ value as in the Cloudnet approach. An additional requirement is that the peak width is not too broad with the maximum peak width at half-height being set to $150 \mathrm{~m}$. This ensures that the identified peak is attenuating rapidly (O'Connor et al., 2004) rather than the relatively weak attenuation expected in precipitation so that threshold exceedance found in precipitation is not enough to trigger false liquid layer identification. The cloud base below the strong $\beta$ value is found using the same method as for the Cloudnet droplet bit algorithm.

Visual validation of our updated algorithm is shown in Fig. 2, which confirms that liquid cloud layer identification during precipitation is more accurate. The Cloudnet processing suite will soon be updated with this new algorithm, which will also improve Cloudnet-derived products. This new algorithm can be used for other applications such as the identification of liquid layers for in-cloud icing detection for wind turbine operators and aviation.

\subsection{Precipitation and fog identification}

In addition to liquid layers, we require fog, precipitation, and ice cloud identification. The profiles in these conditions show particular characteristics (Fig. 1b-d). Precipitation, including ice (we assume that all ice is falling), is identified from 

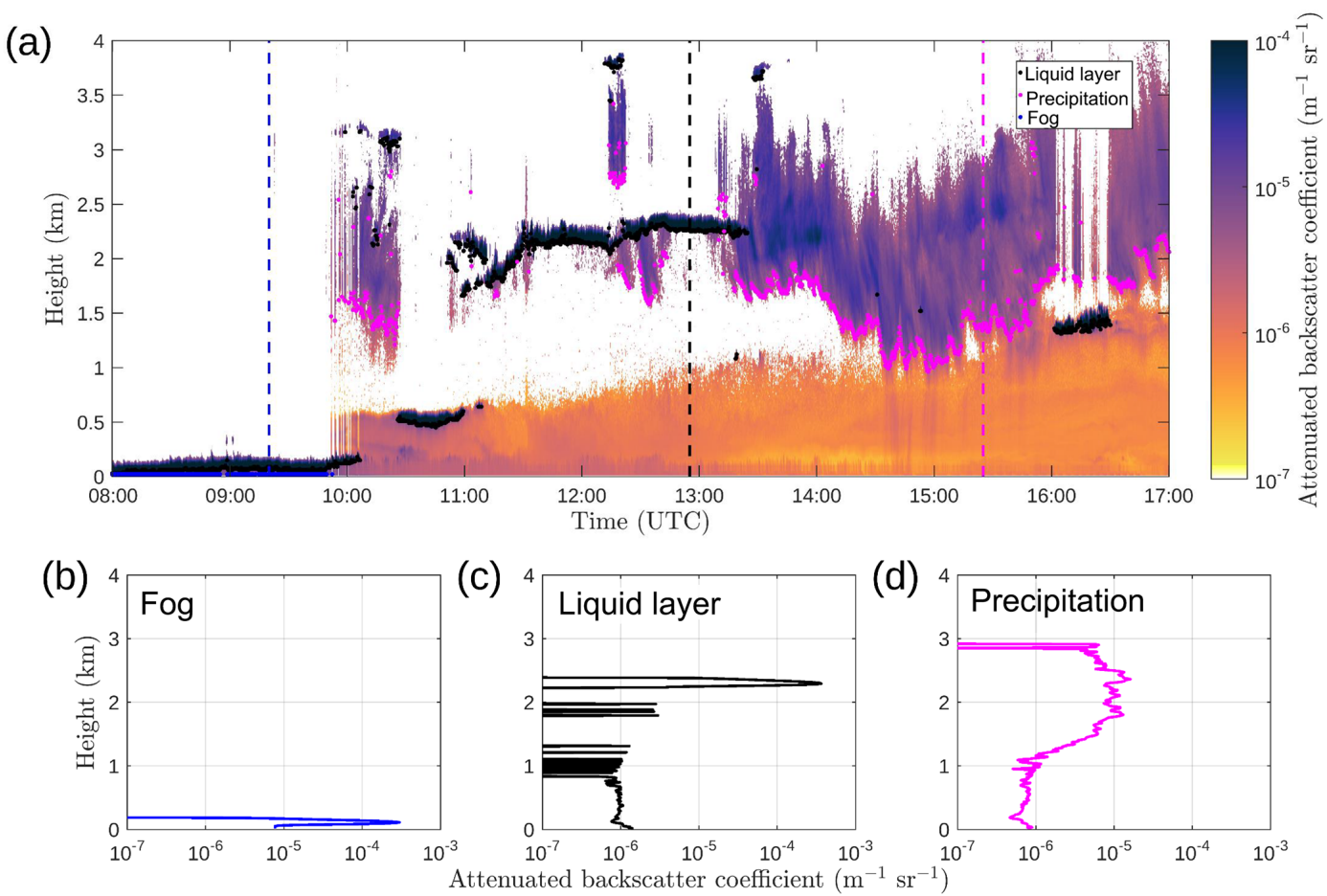

Figure 1. Time-height cross section of attenuated backscatter profiles from a Vaisala CL51 ceilometer on 30 March 2016 at Helsinki, Finland (a). Overplotted are the results from our identification algorithms: fog (blue dots), liquid cloud base (black dots), and precipitation base (magenta dots). Sample attenuated backscatter profiles are also shown for fog (b), liquid cloud layer (c), and precipitation (d). Dashed lines in (a) show the time when the profiles (b)-(d) are measured.

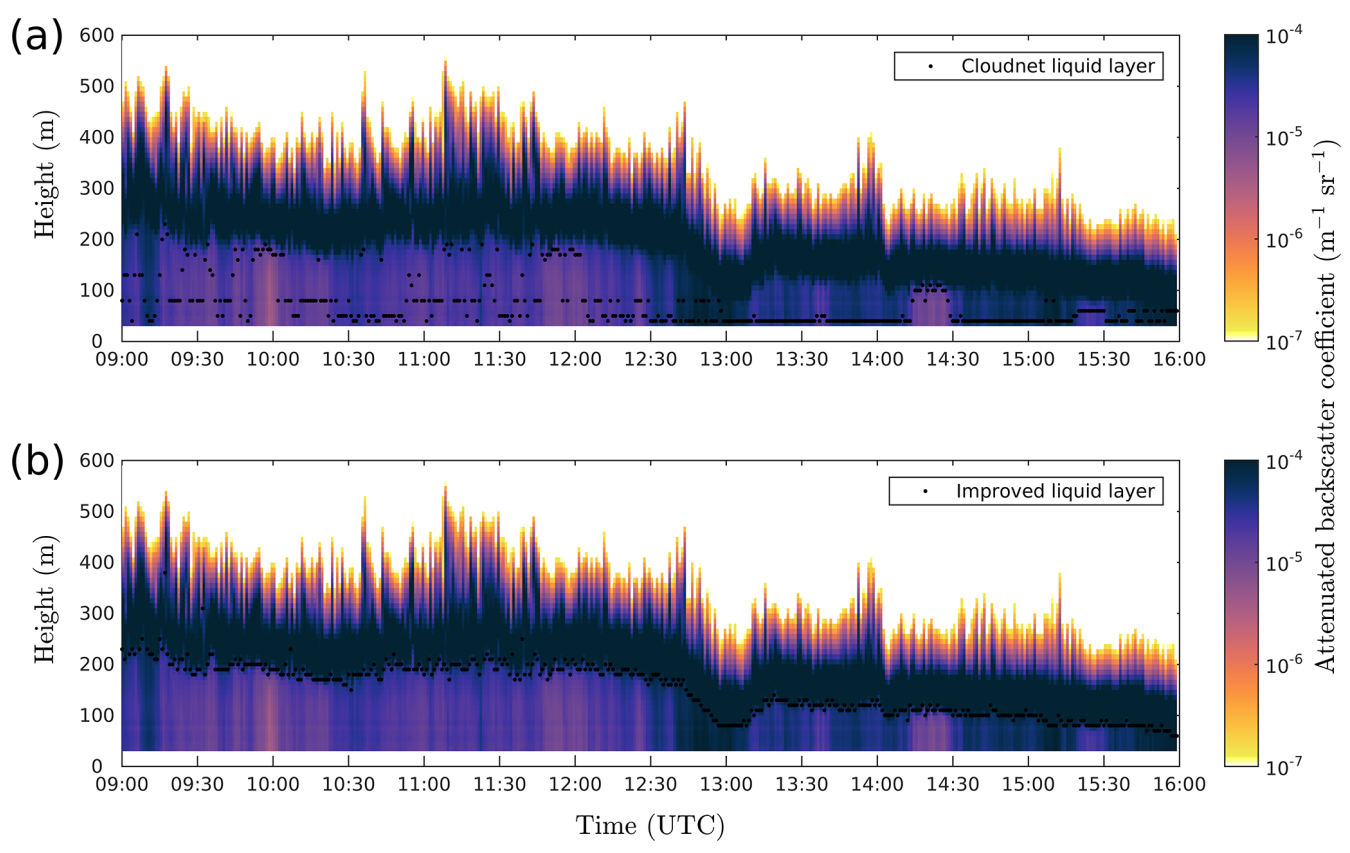

Figure 2. Time-height cross section of attenuated backscatter profiles from a Vaisala CL51 ceilometer on 27 October 2016 at Helsinki, Finland, with the Cloudnet approach (a) and with our updated algorithm (b) for obtaining liquid layer base. A major improvement is seen during precipitation events. 
the shape of the attenuated backscatter profile (Fig. 1d). We identify the base of the precipitating layer, which in practice means the altitude at which the precipitation is either evaporating or reaching the ground. Typically, attenuated backscatter coefficient values are lower for precipitating rain and ice relative to liquid droplets. This is due to their much lower number concentrations even though the particle sizes are larger. The ceilometer signal is not attenuated as rapidly during precipitation and the ceilometer can "see" further into the precipitation. The precipitation algorithm uses a threshold value of $\beta=3 \times 10^{-6} \mathrm{~m}^{-1} \mathrm{sr}^{-1}$, determined to be suitable in this study, together with a layer thickness greater than $350 \mathrm{~m}$ (i.e. the ceilometer backscatter signal is not attenuated within $350 \mathrm{~m}$ ). We determined these thresholds by visual analysis. The layer base is simply the lowest range gate at which these two conditions are satisfied. Both precipitation and a liquid layer can be identified within the same profile.

Fog at the surface cannot always be identified using the liquid layer identification method, which relies on finding a local maximum in the $\beta$ profile. An example of fog is given in Fig. $1 \mathrm{~b}$ in which there are already high $\beta$ values in the first range gate. Here we check the rate of the attenuation above the fog layer maximum as it may not be possible to define a peak. The threshold for fog is set as $\beta=10^{-5} \mathrm{~m}^{-1} \mathrm{sr}^{-1}$, with a $\beta$ value $250 \mathrm{~m}$ above the instrument of $\beta<3 \times 10^{-7} \mathrm{~m}^{-1} \mathrm{sr}^{-1}$.

\section{Model data}

\subsection{The Integrated Forecast System (IFS)}

Forecasts produced by the Integrated Forecast System (IFS), run operationally by the European Centre for MediumRange Weather Forecasts (ECMWF), are analysed in this study. The IFS is a global numerical weather prediction (NWP) system which includes observation processing and data assimilation in addition to the forecast system. The IFS is used to produce a range of different forecasts, from medium-range to seasonal predictions, and both deterministic and ensemble forecasts. In this study we only consider the high-resolution deterministic medium-range forecasts (referred to as HRES) which have a horizontal resolution of approximately $9 \mathrm{~km}$ and 137 vertical levels. The vertical grid spacing is non-uniform and below $15 \mathrm{~km}$ varies from 20 to $300 \mathrm{~m}$ with higher resolution closer to the ground. The temporal resolution of the model output is $1 \mathrm{~h}$ and forecasts up to 10 days in length are run every $12 \mathrm{~h}$. A full description of the IFS can be found from ECMWF documentation: https: //www.ecmwf.int/en/forecasts/documentation-and-support/ changes-ecmwf-model/ifs-documentation (last access: 1 November 2018).

The IFS is under constant development and typically a new version becomes operational every 6-12 months. Therefore, unlike reanalysis, which is based on a static model sys- tem, the archived forecasts from the operational IFS reflect changes in the model. Although the aim of this paper is not to quantify how changes to the IFS affect the cloud and solar radiation forecasts, a brief overview of model updates is given here.

Several upgrades have been implemented into the IFS during the 4-year (2014-2017) data period that is used in this study (all are described in the IFS documentation: https: //www.ecmwf.int/en/forecasts/documentation-and-support/ changes-ecmwf-model/ifs-documentation.) A major upgrade occurred in March 2016 when the horizontal grid was changed from a cubic-reduced Gaussian grid to an octahedral-reduced Gaussian grid, resulting in an increase in horizontal resolution from 16 to $9 \mathrm{~km}$. The cloud, convection, and radiation parameterization schemes strongly influence the forecast of clouds and radiation and all of these schemes have undergone updates during the 4-year period considered here. Notably, the radiation scheme was updated from the McRad scheme (Morcrette et al., 2008 ) to the scientifically improved and computationally cheaper ECRAD scheme (Hogan and Bozzo, 2016) in 2016. Aerosols also impact radiation forecasts and are represented in the IFS by a seasonally varying climatology. In July 2017 the aerosol climatology was updated to one derived from the aerosol model developed by the Copernicus Atmospheric Monitoring Service and coupled to the IFS (Bozzo et al., 2017). Note that in the current version of the IFS aerosol and clouds do not interact.

\subsection{Model output used in this study}

We use day-ahead forecasts, which have been initialized at 12:00 UTC the previous day and correspond to forecast hours $t+12$ to $t+35$, obtained from the closest land grid point to the measurement site $2.1 \mathrm{~km}$ away. Day-ahead forecasts are commonly used in the solar energy field for estimating daily production for the energy market. A list of the model variables we use is given in Table 1.

One goal is to develop simple and robust methods for evaluating the skill that the model has in forecasting clouds and solar radiation, which can be rapidly applied to numerous sites globally. Therefore, we take the single-level cloud forecast variables: low cloud cover (LCC) and medium cloud cover (MCC). These are defined in the IFS as follows: low is model levels with a pressure greater than 0.8 times the surface pressure (from the ground to approximately $2 \mathrm{~km}$ in altitude); medium encompasses model levels with a pressure between 0.45 and 0.8 times surface pressure (approximately 2-6 km). For IFS, the cloud layer overlap is also taken into account when calculating LCC and MCC, and the degree of randomness in cloud overlap is a function of the separation distance between layers (the greater the distance between layers, the more randomly overlapped they are; Hogan and Illingworth, 2000). For solar radiation forecasts, we use the surface solar radiation downward (SSRD), which is a single- 
Table 1. ECMWF IFS model variables. Model-level fields have a vertical dimension. Single-level fields have no vertical dimension; this includes surface fields and column-integrated fields. Obtained via the Meteorological Archival and Retrieval System (MARS) at ECMWF using a grid resolution of $0.125^{\circ}$.

\begin{tabular}{lllll}
\hline Variable & Short name & Unit & Variable type & Other \\
\hline Low cloud cover & LCC & $0-1$ & single level & instant \\
Medium cloud cover & MCC & $0-1$ & single level & instant \\
Specific cloud liquid water content & CLWC & $\mathrm{kg} \mathrm{kg}^{-1}$ & model level & instant \\
Temperature & $T$ & $\mathrm{~K}$ & model level & instant \\
Pressure & PRES & $\mathrm{Pa}$ & model level & instant \\
Surface pressure & SP & $\mathrm{Pa}$ & single level & instant \\
Surface solar radiation downward & SSRD & $\mathrm{J} \mathrm{m}^{-2}$ & single level & cumulative \\
TOA incident solar radiation & TISR & $\mathrm{J} \mathrm{m}^{-2}$ & single level & cumulative \\
\hline
\end{tabular}

level parameter output hourly as an accumulated value (from the start of the forecast) in units of $\mathrm{J} \mathrm{m}^{-2}$.

Other model variables are also downloaded for further calculation and for more detailed investigation of the sources of forecast error. Pressure (PRES) on model levels and surface pressure (SP) are used to determine the altitude levels for low and medium cloud cover classes for ceilometer data post-processing. Temperature ( $T$ ) on model levels is used for classifying warm and cold (supercooled) liquid clouds. Specific cloud liquid water content on model levels (CLWC), provided as a mixing ratio, is used to calculate the total cloud liquid water path (LWP).

\section{Methods for evaluating the model performance}

Some further calculation is needed in order to evaluate the model output against the observations, as the variables obtained from the model and observations are not directly comparable. The forecast cloud cover is a single-level variable representing instantaneous values of column-integrated cloud coverage over an area (model grid of approximately $16 \times 16 \mathrm{~km}$ before the resolution upgrade and $9 \times 9 \mathrm{~km}$ area after the resolution upgrade) with hourly resolution. The ceilometer attenuated backscatter profile observations are point measurements with high temporal resolution $(15 \mathrm{~s})$, from which cloud occurrence can be derived. The forecast solar radiation is an accumulated value in $\mathrm{Jm}^{-2}$ since the beginning of the forecast, whereas the observed GHI (in $\mathrm{W} \mathrm{m}^{-2}$ ) is a point measurement averaged to $1 \mathrm{~min}$ resolution. Post-processing of both forecast and observations is required to obtain a comparable dataset, as discussed in the following subsections. After further post-processing, skill scores are then used to evaluate the cloud cover forecasts, and different error metrics are used to calculate the solar radiation forecast error.

In this study, we only consider daytime hours for model evaluation as our focus is on solar radiation forecasts. Therefore, hours with hourly-averaged GHI measurements less than $5 \mathrm{~W} \mathrm{~m}^{-2}$ are removed. For northern latitudes, this re- sults in a range from 2 to $19 \mathrm{hday}^{-1}$, depending on the season (short days in winter and long days in summer). Furthermore, it is required that the data availability of observations over each hour is at least $75 \%$; otherwise the hour is discarded from the analysis.

\subsection{Post-processing of cloud cover forecasts and ceilometer observations}

The difference arising from the fundamental differences in cloud information obtained from the model (grid value) and observations (point measurement) must be compensated for. As the clouds are advected over the measurement site, the temporal average of the point measurements of cloud occurrence is correlated with the cloud cover over an area. Therefore, averaging the ceilometer observations over a certain time window is assumed to correspond to cloud cover represented in grid space. The suitable averaging time window for cloud cover may not be easy to define; here $1 \mathrm{~h}$ averages are used as this is the temporal resolution of the model output. The horizontal resolution of the model is $16 \mathrm{~km} / 9 \mathrm{~km}$, and therefore $1 \mathrm{~h}$ averaging corresponds to advection speeds of 4.5 or $2.5 \mathrm{~m} \mathrm{~s}^{-1}$. However, we are aware that this averaging procedure may not always be appropriate for comparison and is kept in mind when analysing the results.

High and thin ice clouds are not reliably detected with ceilometers (see Sect. 2), and therefore we only consider clouds at low to medium altitudes in both the model and observations. We do not evaluate the model total cloud cover (TCC), as this contains contributions from high clouds.

The model variables LCC and MCC account for cloud within their relevant height ranges regardless of whether there is cloud in a lower level. In contrast, the ceilometer usually only detects the base of the first cloud layer. For example, the ceilometer may detect a cloud base to be below $2 \mathrm{~km}$, hence defining it as low cloud, but the cloud may also contribute significantly to mid-level cloud cover, which is not captured by the ceilometer. In strong precipitation, the lidar signal may be sufficiently attenuated so that the liquid cloud base can no longer be detected above the precipitation. In 
these cases, the bottom of the precipitation layer is treated as a cloud base, even though in reality the cloud producing the rain is at higher altitude. Thus, we combine low and medium cloud cover, rather than investigating them separately.

Cloud cover is estimated from the ceilometer data as follows: first, the attenuated backscatter profiles are averaged over $1 \mathrm{~min}$ before applying the algorithms described in Sect. 3. Then, liquid layers, precipitation (including ice clouds), and fog are identified for each 1 min profile. The forecast pressure on model levels is interpolated to the ceilometer range gate heights using the model height (ECMWF uses a terrain-following eta coordinate system). Cloud cover at each level (low and medium, defined in terms of pressure as for the model) is calculated as the percentage of cloud occurrence (occurrence of liquid cloud, precipitation or ice cloud, or fog) within each level over each hour. Finally, the observed cloud cover is the hourly sum of the observed low and medium cloud cover. Note that here the observed cloud cover is a summation since it is calculated from time series of independent columns for which only the first cloud layer contributes to the cloud cover calculation (the lowest layer).

The forecast LCC and MCC represent the fractional cloud cover (from 0 to 1 ) over the grid point, and combining these requires an assumed overlap factor. In this study we use the random overlap assumption, which may result in a slight overestimate (Hogan and Illingworth, 2000).

\subsection{Post-processing of solar radiation forecasts and solar radiation observations}

Forecast surface solar radiation (SSRD) is compared against the observed global horizontal irradiance (GHI). Values of SSRD require de-accumulating to hourly averages as the forecast solar radiation is an accumulated field from the beginning of the forecast and is transformed from $\mathrm{Jm}^{-2}$ to $\mathrm{Wm}^{-2}$. Observed $1 \mathrm{~min}$ averaged GHI measurements $\left(\mathrm{W} \mathrm{m}^{-2}\right)$ are averaged over $1 \mathrm{~h}$ for comparison. It should be noted that the model radiative transfer scheme is unlikely to completely account for the three-dimensional nature of radiative transfer as experienced by the observations.

\subsection{Skill scores for cloud cover forecasts and error metrics for solar radiation forecast error}

Cloud cover forecasts are evaluated with 2-D histograms and skill scores. We use the mean absolute error skill score (MAESS; Hogan et al., 2009) and mean squared error skill score (MSESS; Murphy, 1988), which compare the occurrence of a cloud separately in observations and in forecasts, and take into account the magnitude of the difference. MAESS uses the absolute difference between the forecast and observed value, and MSESS uses the squared difference, which for two forecasts with the same absolute error will penalize the forecast with one or two large errors more than the

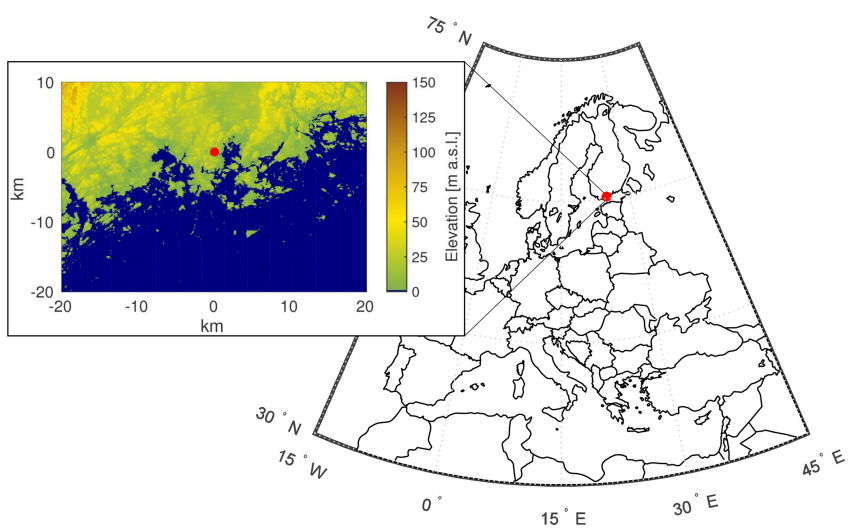

Figure 3. Measurement site at Helsinki, Finland $\left(60.204^{\circ} \mathrm{N}\right.$, $\left.24.961^{\circ} \mathrm{E}\right)$.

forecast with many small errors. The skill scores are based on the contingency table (Table A1 in Appendix A1), in which the occurrence of hits, false alarms, misses, and correct negative values by a given cloud cover threshold are calculated. For example, a hit occurs when both forecast and observed cloud cover are above a given cloud cover threshold. Here, the threshold for cloud cover is set to 0.05 , following the method used by Hogan et al. (2009). Therefore, a hit means that some amount of cloud is both forecast and observed; however, a hit does not yet imply a perfect forecast. For both MAESS and MSESS, the skill of a random forecast is 0 and a perfect forecast 1 . The equation for MAESS and MSESS is given in Appendix A1.

The error metrics mean absolute error (MAE), mean absolute percentage error (MAPE), mean error or bias (ME), and root mean square error (RMSE) are used to evaluate the solar radiation forecast errors. These error metrics are defined in Appendix A2. MAE, RMSE, and ME are absolute error metrics and result in forecast error in $\mathrm{W} \mathrm{m}^{-2}$, whereas MAPE is a relative error given in percent. ME is the only error metric that shows the sign of the error. A positive bias is seen when the model overestimates the incoming surface solar radiation, whereas a negative bias is when the model underestimates the incoming solar radiation.

\section{Site characteristics and cloud and radiation climatology}

The measurement site is located on the roof of FMI in Helsinki, Finland $\left(60.204^{\circ} \mathrm{N}, 24.961^{\circ} \mathrm{E}\right.$; Fig. 3, measurements at $26 \mathrm{~m}$ above sea level), located less than $10 \mathrm{~km}$ from the coastline of the Gulf of Finland. Coastal effects, such as sea breezes, are common. There are no large variations in topography around the site.

We investigate the cloudiness and solar resource at this site using 4 years of ceilometer observations and solar radiation measurements. There is an annual variation in the 
(a)
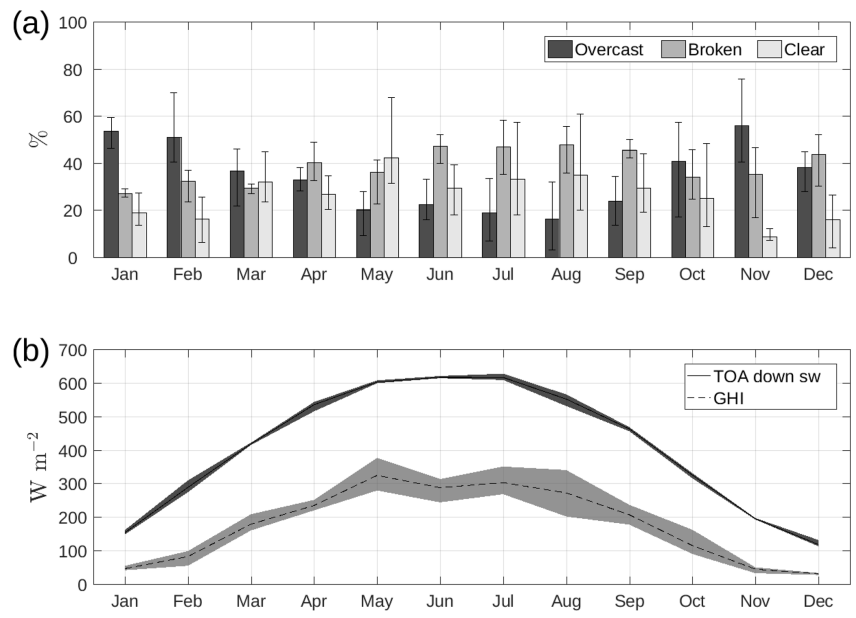

Figure 4. Relative occurrence of overcast, broken cloud, and clearsky conditions (a). Bars show yearly variation (min, max). Annual variation in observed GHI and forecast top-of-atmosphere downwelling shortwave radiation (b). Shaded area represents the yearto-year variation in monthly means.

observed cloudiness at the site (Fig. 4a) with overcast conditions (cloud cover $\geq 0.95$ ) being more common in winter and less common in summer. In contrast, broken cloud $(0.05<$ cloud cover $<0.95)$ and clear (cloud cover $\leq 0.05)$ conditions are most common in summer and least common in winter. The variation in cloudiness is quite high from year to year, especially in summer, but in winter the most probable sky condition contains cloud.

In addition to the observed annual variation of cloudiness, the observed annual variation of incoming solar radiation is strongly influenced by the northern location of the site $\left(60^{\circ} \mathrm{N}\right)$. Due to the change in the solar zenith angle, the length of the shortest day of the year (winter solstice on 21 or 22 December) is less than $6 \mathrm{~h}$ and the length of the longest day (summer solstice between 20 and 22 June) is almost $19 \mathrm{~h}$. The amount of solar radiation at the top of the atmosphere is much higher during summer when the solar zenith angle is also much higher (Fig. 4b, solid line). This signal is also clear in the amount of solar radiation reaching the ground, the measured GHI (Fig. 4b, dashed line), which is dependent on both the incoming solar radiation at the top of atmosphere and the attenuation of the downward flux due to clouds, aerosols, and atmospheric gases. The year-to-year variation in the monthly mean of measured GHI is much greater during summer months (lighter shaded area in Fig. 4b), with variations reaching $140 \mathrm{~W} \mathrm{~m}^{-2}$ in August, which is larger than the monthly mean GHI during winter months.

To investigate the seasonal variation, we define seasons based on the annual variation in the solar resource (Fig. 4b). The summer season is defined as May to July when the solar resource is at a maximum, and winter is defined as November to January when the solar resource is at a minimum (spring is February to April, autumn is August to October).

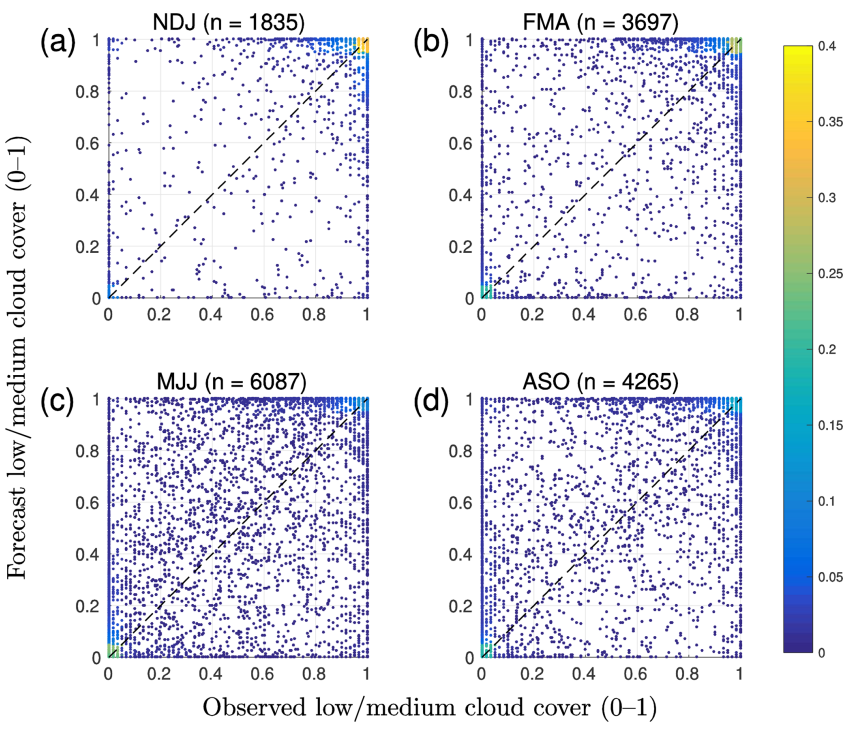

Figure 5. Seasonal normalized density scatter plots of observed and forecast cloud cover (total counts for each season are given in the titles). Seasons are defined based on the annual distribution of incoming solar radiation: (a) winter (November to January), (b) spring (February to April), (c) summer (May to July), and (d) autumn (August to October).

\section{Forecast skill in predicting clouds and radiation}

\subsection{How well are clouds forecast?}

We now investigate how well the IFS forecasts clouds over our site in Helsinki, Finland. Since we are interested in the solar resource we only evaluate time steps in which the hourly-averaged observed GHI is greater than $5 \mathrm{~W} \mathrm{~m}^{-2}$ to link the skill in forecasting clouds to the skill in forecasting radiation (Sect. 7.3).

In Fig. 5 we compare the observed and forecast cloud cover for each season. For a perfect forecast, all values would lie on the diagonal (dashed line) in each scatter plot. For all seasons, the majority of cloud cover values are concentrated around clear conditions (pair $0 ; 0$ ) and overcast conditions (pair $1 ; 1$ ) for both observations and forecasts. This suggests that not only are clear and overcast conditions the most commonly observed, but also most skilfully forecast in all seasons. During winter the vast majority of cloud cover observations and forecasts are at (or close to) overcast (Fig. 5a). Clear-sky conditions are more common in other seasons (both observations and model). The large spread for both observed and forecast cloud cover values between 0.1 and 0.9 indicates that partly cloudy conditions are challenging for the IFS to correctly predict. However, these cases are not as common as clear and overcast cases, which is a result of observed and forecast cloud cover distributions being strongly U-shaped for typical NWP model grid sizes (Hogan et al., 2009; Mittermaier, 2012; Morcrette et al., 2014). It is 

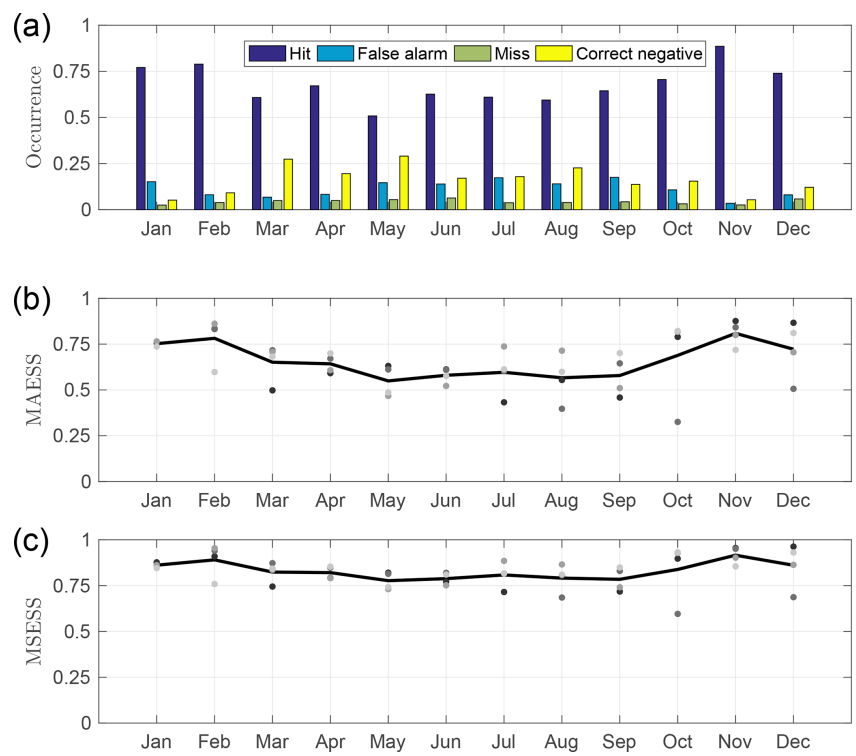

Figure 6. Relative occurrence of elements in the contingency table (hit, false alarm, miss, and correct negative) for each month with a cloud cover threshold of 0.05 (a). Monthly mean skill scores for cloud cover: MAESS (b) and MSESS (c), individual monthly mean for each year (dots), and 4-year average (line).

also notable that, during all seasons, there are values on the boundaries of the scatter plot away from the diagonal, for example, where the model is incorrectly forecasting clear sky during cloudy conditions or overcast conditions during clear or broken skies. Summer and autumn seasons (Fig. 5c, d) display more broken cloud conditions, as also seen in Fig. 4a, when the solar resource is high (Fig. 4b).

Skill scores represent the model's ability to forecast a given variable. To calculate skill scores, we generate a contingency table for cloud cover. This requires a binary forecast so we use a threshold cloud cover value of 0.05 as in Hogan et al. (2009) to define the presence of cloud: a hit is cloud observed and forecast; a false alarm is cloud not observed but forecast; a miss is cloud observed but not forecast; and a correct negative is cloud not observed or forecast.

The annual relative occurrences of contingency table elements (hit, false alarm, miss, correct negative) are shown in Fig. 6a. During all months, hit has the highest relative occurrence (mean $68 \%$ ), indicating that the model usually contains some low or mid-level cloud when cloud is also observed at these levels. The hit occurrence is greatest between October and February when overcast conditions are also most common (Fig. 4a). Note that a hit requires that both observations and model have some cloud, but it does not necessarily represent a perfect forecast. Similarly, the relative occurrence of correct negative is highest during spring and summer months. False alarms are most common in summer and autumn when their relative occurrence reaches $17 \%$.
The relative occurrence of missed clouds is low (mean $4 \%$ ) for all months and there is no clear seasonal cycle.

Skill scores are then generated from the contingency table; we use MAESS and MSESS as these take into account the magnitude of the difference between the observed and forecast cloud cover (Fig. 6b, c). MAESS and MSESS both show annual variation, being highest during winter months and lowest during summer months. This information is important, especially for solar energy purposes, as it shows that clouds are forecast less skilfully in summer, which is when the solar resource is greatest. There are also notable variations in skill scores from year to year, especially in October and December. MSESS is greater than MAESS, especially during summer when more broken cloud conditions are expected.

\subsection{How well is solar radiation forecast?}

As expected, there is a large seasonal variation in observed GHI: up to $900 \mathrm{~W} \mathrm{~m}^{-2}$ in summer (Fig. 7c) and less than $300 \mathrm{~W} \mathrm{~m}^{-2}$ in winter (Fig. 7a). The absolute error in the solar radiation forecast can therefore potentially be much higher in summer and is evident in the potential range of scatter between observed GHI and forecast GHI for each season (Fig. 7). The forecast of solar radiation is usually overestimated in all seasons (Fig. 8), especially for low irradiance values for which the positive bias is more obvious. Solar radiation forecast MAE (Fig. 8a, solid line) is greater in summer than in winter, as is the year-to-year variation in monthly absolute errors (shaded area in Fig. 8a). There is no clear seasonal cycle in the variation in the relative error (MAPE) from year to year; however, MAPE itself peaks in February and November.

The mean error (ME) in the solar radiation forecast is positive when the model overestimates solar radiation at the surface. Figure $8 \mathrm{~b}$ shows separate calculations of the monthly mean positive (red) and negative (blue) bias in forecast GHI. Throughout the year, the positive bias (both absolute and relative) is greater than the negative bias, and thus the model overestimates solar radiation more than it underestimates. The year-to-year variation in relative positive bias is also larger than the relative negative bias. For example, the relative positive bias in solar radiation forecast ranges between $50 \%$ and $125 \%$, whereas the relative negative bias is rather constant at around $25 \%$. Overestimates are also more common than underestimates (not shown). The result is an overall positive bias in forecast GHI. Both positive bias metrics (relative and absolute) show the same seasonal response as the corresponding MAE/MAPE metric and the negative bias metric shows the same summer enhancement as the positive bias but with the opposite sign. 

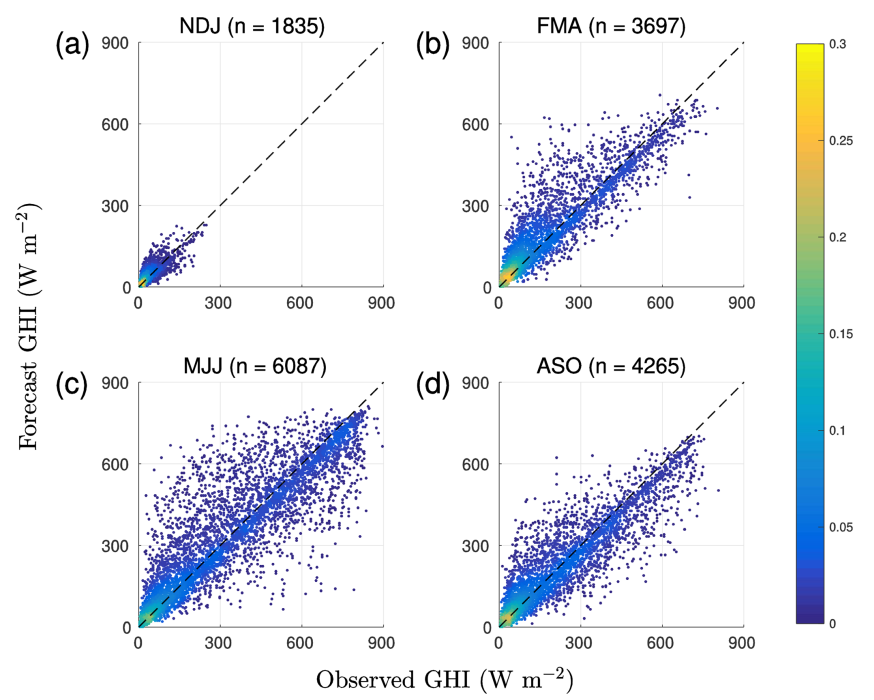

Figure 7. Seasonal normalized density scatter plots of observed and forecast GHI (total counts for each season are given in the titles). Seasons are defined based on the annual distribution of incoming solar radiation: (a) winter (November to January), (b) spring (February to April), (c) summer (May to July), and (d) autumn (August to October).
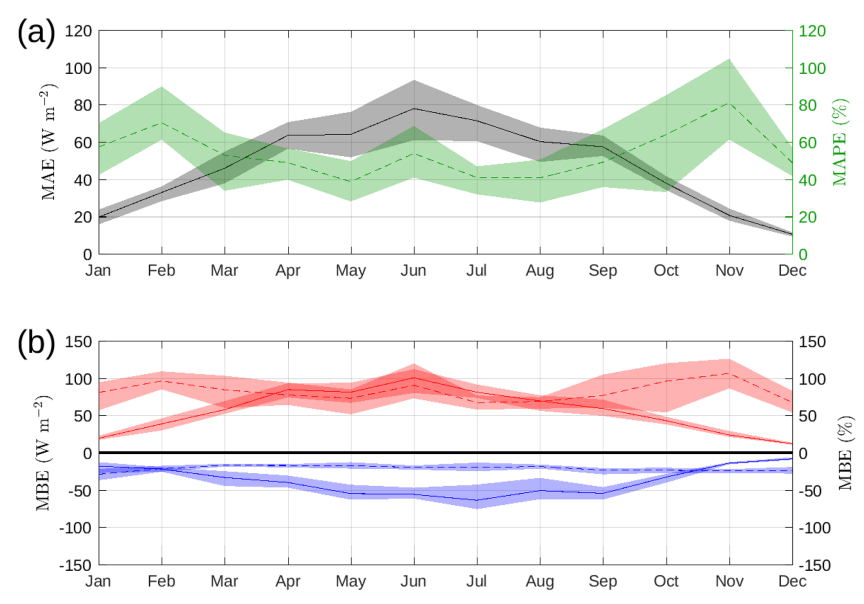

Figure 8. Monthly MAE (black solid line) and MAPE (green dashed line) in solar radiation forecast (a). Monthly absolute (solid line) and relative (dashed line) ME (b). Positive bias (red) and negative bias (blue) are shown separately; shaded area represents yearto-year variation.

\subsection{How do errors in cloud cover impact the solar radiation forecast?}

Assuming the correct representation of radiative transfer in the atmosphere, with only the forecast of cloud impacting the solar radiation forecast at the surface (no change in aerosol or humidity), then an increase in forecast cloud cover would be expected to result in a reduction in the amount of forecast solar radiation. However, the amount of cloud may be
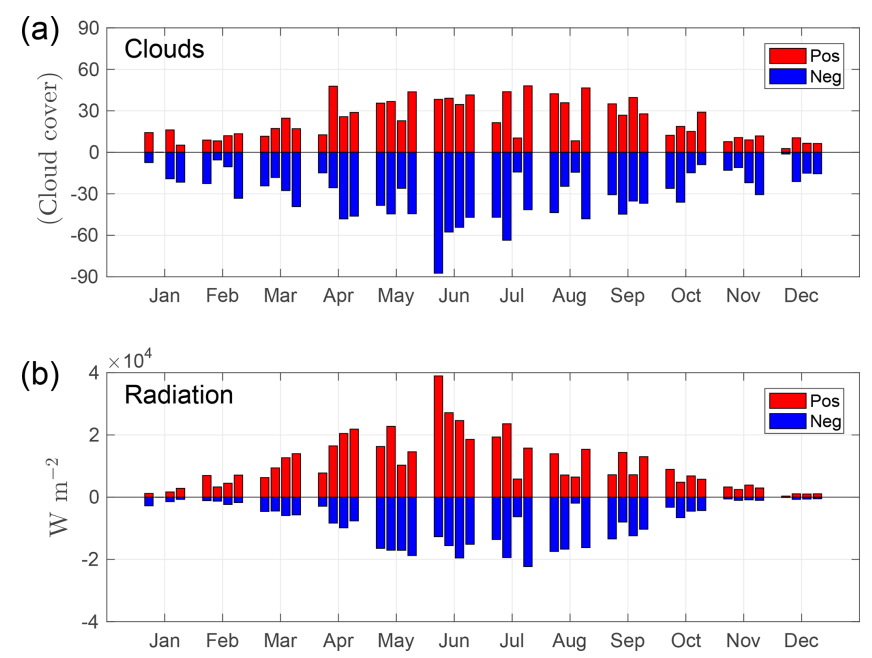

Figure 9. Monthly accumulated positive (red) and negative (blue) bias in cloud cover forecast (a) and solar radiation forecast (b). The four bars in each month represent individual years (2014-2017).

correctly forecast, but not the cloud properties. Since cloud properties are directly responsible for the cloud radiative effect, both cloud amount and properties should be correctly forecast in order to obtain a reliable solar radiation forecast.

Figure 9 shows the annual cycle of accumulated positive and negative bias in the cloud cover forecast and solar radiation forecast. It can be seen that months with a large accumulated negative bias in cloud cover forecasts (e.g. June 2014) show a notably large accumulated positive bias in the solar radiation forecast. However, not all months show a clear correlation between a negative bias in the cloud cover forecast and a positive bias in the solar radiation forecast. This is most probably due to compensating effects whereby, for example, the cloud cover forecast could be overestimated (positive bias in cloud cover) but the liquid water content forecast is underestimated (which would result in positive bias in solar radiation forecasts).

To investigate how well the forecast cloud cover corresponds to the observed cloud cover, the counts of hourly observed and forecast cloud cover values are paired together in 2-D histograms (Fig. 10a). For perfect forecasts, all counts would lie on the diagonal. Figure 10a shows that there are many correctly forecast situations for clear sky $(0 ; 0)$ and overcast $(1 ; 1)$. However, it is clear that there are many values on the boundaries, which means that cloud is either observed and not forecast (miss) or cloud is forecast but not observed (false alarm). At $1 \mathrm{~h}$ resolution, $47 \%$ of the total number of counts is above the diagonal, and thus the forecast cloud cover is overestimated on average. The forecast underestimates cloud cover $34 \%$ of the time. Note that changing the overlap assumption from random to maximum when calculating the combined cloud cover $(\mathrm{LCC}+\mathrm{MCC})$ changes these values by $3 \%$. 


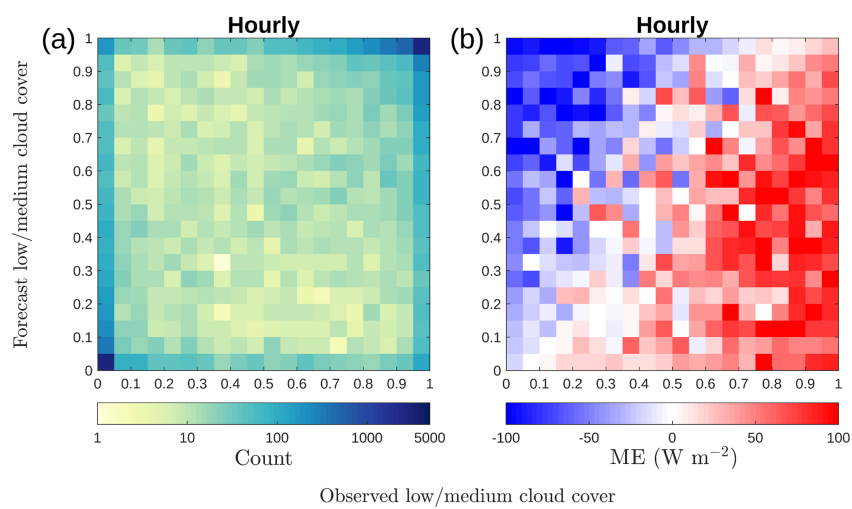

Figure 10. 2-D histogram of observed and forecast cloud cover (a), with colours representing counts on a logarithmic scale, and ME in solar radiation forecast (b) for each cloud cover pair in (a).

The solar radiation forecast ME for concurrent pairs of cloud cover values in Fig. 10a is presented in Fig. 10b. ME values below the diagonal, for which the forecast cloud cover is underestimated, are mostly positive; similarly, ME values above the diagonal are mostly negative, and the forecast cloud cover is overestimated. Note that the change from positive to negative ME does not quite follow the diagonal, with minimal bias appearing to follow a line from $(0.1 ; 0)$ to $(0.8 ; 1)$; i.e. observed cloud cover greater than 0.9 shows a positive solar radiation forecast $\mathrm{ME}\left(27 \mathrm{~W} \mathrm{~m}^{-2}\right)$ and observed cloud cover less than 0.1 shows negative ME $\left(-16 \mathrm{~W} \mathrm{~m}^{-2}\right)$. This negative bias during clear-sky situations over Helsinki was also observed by Rontu and Lindfors (2018) and is most likely due to the aerosol climatology implemented in the model having too much aerosol. Another possible source of negative bias during clear-sky situations would be too much water vapour in the atmosphere. There are earlier studies showing similar results elsewhere (Ahlgrimm and Forbes, 2012; Frank et al., 2018). Overcast situations occur more frequently than clear sky ( $23 \%$ of the time), resulting in the overall positive bias in the solar radiation forecast.

\section{Impact of temporal averaging}

Forecasting individual clouds in the right place at the right time is challenging and here we investigate whether temporal averaging improves the cloud forecast and therefore the radiation forecast. Different averaging windows (3-hourly, 6hourly, 12-hourly, daily) are used in preparing the data for evaluation in the same manner as for Fig. 10a and the results for selected averaging windows are shown in Fig. 11a-c. The agreement between observed and forecast cloud cover improves with increasing averaging windows, and the number of cases of extreme misses and false alarms (corners $(1 ; 0)$ and $(0 ; 1))$ decreases.
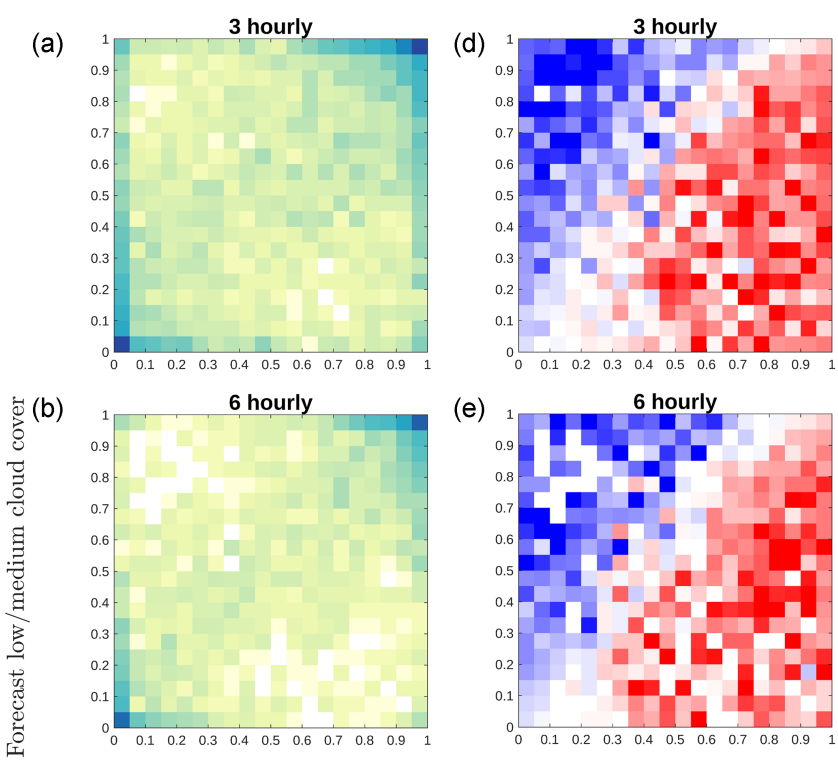

(c)
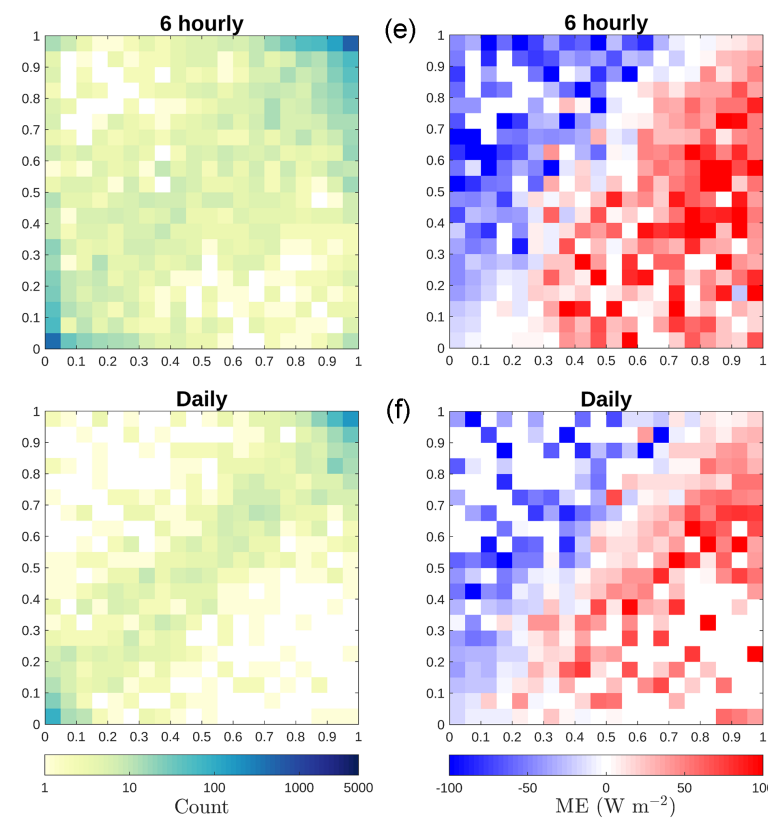

(f)

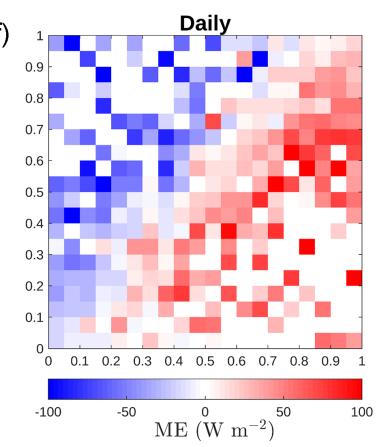

Observed low/medium cloud cover

Figure 11. Same plots as Fig. 10, except for different averaging time windows (3 hourly, 6 hourly, and daily). 2-D histograms of observed and forecast cloud cover $(\mathbf{a}-\mathbf{c})$, with colours representing counts on a logarithmic scale, and $\mathrm{ME}$ in solar radiation forecast (d-f) for each cloud cover pair in (a-c).

When calculated separately, the magnitudes of the positive and negative solar radiation forecast biases for concurrent pairs of cloud cover values decrease with increasing averaging time. The mean positive bias decreases from $65 \mathrm{~W} \mathrm{~m}^{-2}$ when averaging over $1 \mathrm{~h}$ to $35 \mathrm{~W} \mathrm{~m}^{-2}$ when averaging over 1 day, and the mean negative bias reduces from -46 to $-27 \mathrm{~W} \mathrm{~m}^{-2}$. The overall bias remains around $8 \mathrm{~W} \mathrm{~m}^{-2}$. Increasing the averaging window does not alter the pattern in which the change from positive to negative $\mathrm{ME}$ is away from the diagonal. The negative bias in clear-sky conditions and positive bias in overcast conditions are still present, suggesting that the bias is likely to be due to cloud properties rather than the cloud presence.

Figure 12 summarizes the impact of temporal averaging on the skill in forecasting cloud cover and the error in forecasting solar radiation, with skill clearly increasing and error decreasing as the averaging window is lengthened. Extreme misses and false alarms for cloud cover are reduced, and for 

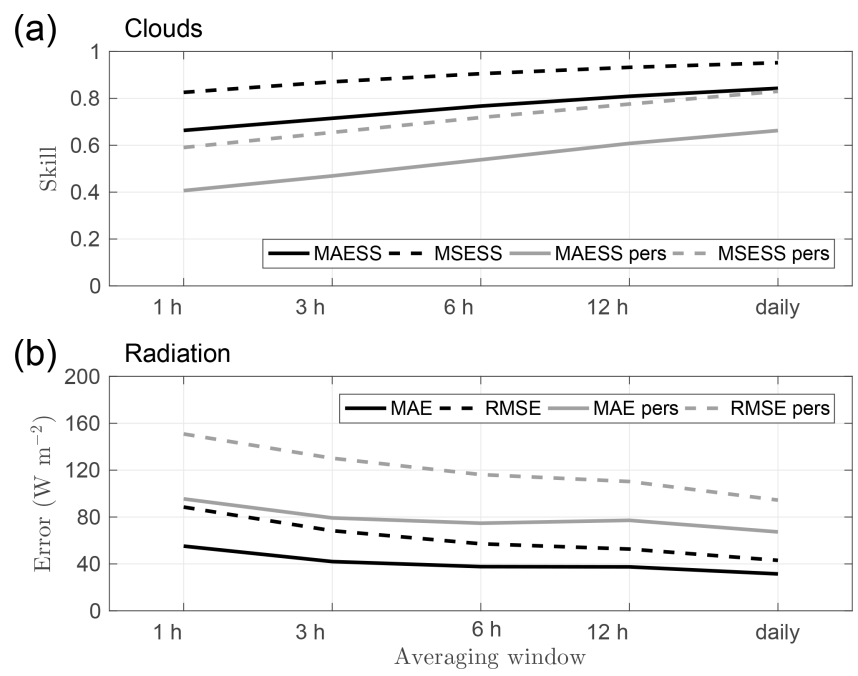

Figure 12. Cloud cover forecast skill scores (a) and error in solar radiation forecast (b) for different averaging time windows, including persistence forecasts (grey lines). Note the non-linear $x$ axis.

GHI MAE, the individual absolute errors are reduced with temporal averaging. Persistence forecasts were also investigated; a persistence forecast uses the hourly forecast values from the day before. The skill for the cloud cover persistence forecast also increases with increasing temporal averaging, as does the reduction of error in the persistence GHI forecast; however, these are not as good as the actual forecasts at this location.

\section{Overcast analysis}

Figures 10 and 11 show a positive bias in the solar radiation forecast, even when overcast conditions are correctly forecast, for all averaging windows. As the cloud amount is correctly forecast, this suggests that the bias must be due to cloud properties. We investigate the forecast cloud base temperature and cloud liquid water path (LWP). Previous studies have shown that clouds containing supercooled liquid $\left(T<0{ }^{\circ} \mathrm{C}\right)$ are poorly forecast (Forbes and Ahlgrimm, 2014; Barrett et al., 2017), and LWP is one parameter that contains information on the amount of liquid water in a cloud, directly impacting how much solar radiation is transmitted through the cloud.

We consider correctly forecast overcast cases (observed and forecast cloud cover $>0.9$ ) containing liquid. The clouds are classified as warm or cold (supercooled), depending on their cloud base temperature, using the temperature profile from the IFS as no observed temperature profiles are available. We then bin the clouds based on their forecast cloud LWP obtained by integrating the forecast cloud liquid water content (CLWC; Table 1). We selected three bins representing relatively high (LWP $>0.2 \mathrm{~kg} \mathrm{~m}^{-2}$ ), moderate $\left(0.2 \mathrm{~kg} \mathrm{~m}^{-2} \geq \mathrm{LWP} \geq 0.05 \mathrm{~kg} \mathrm{~m}^{-2}\right)$, and low $(\mathrm{LWP}<$
$0.05 \mathrm{~kg} \mathrm{~m}^{-2}$ ) cloud liquid water content. These values were selected based on the range of optical depths that would be expected for each LWP range bin. Unfortunately, there was no observed LWP available for this measurement site.

Figure 13 shows that the positive bias in the solar radiation forecast increases with decreasing LWP. Note that the response is similar for both warm and cold liquid clouds. For warm clouds, the ME in GHI increases from $16 \mathrm{~W} \mathrm{~m}^{-2}$ for clouds with high LWP to $70 \mathrm{~W} \mathrm{~m}^{-2}$ for clouds with low LWP. For cold clouds, the ME in GHI increases from $15 \mathrm{~W} \mathrm{~m}^{-2}$ for clouds with high LWP to $36 \mathrm{~W} \mathrm{~m}^{-2}$ for clouds with low LWP. This suggests that either forecast clouds do not have enough LWP or that the optical properties of clouds with low LWP are not properly modelled. The first conclusion, that forecast clouds do not have enough LWP, is consistent with the findings of Ahlgrimm and Forbes (2012). They found a positive radiation bias in ECMWF IFS for overcast situations with low cloud at the Atmospheric Radiation Measurement site in the Southern Great Plains. Furthermore, they found that IFS overestimates the occurrence of clouds with low LWP and underestimates the number of clouds with high LWP, which also results in a positive bias in solar radiation forecasts. Challenges in correctly modelling supercooled liquid clouds have previously been reported, but our results suggest that the issue of a positive bias in GHI is more pronounced for warm clouds and not just an issue for supercooled liquid clouds.

Also of interest is that the relative bias in $\mathrm{GHI}$ is constant across a wide range of GHI values. This implies that a simple LWP-dependent correction factor could be applied to the GHI forecast to remove the bias.

\section{Conclusions}

We have used ceilometer and solar radiation measurements to evaluate the cloud cover and solar radiation forecasts in the ECMWF operational IFS model over Helsinki, Finland. To obtain reliable cloud cover information from the ceilometer attenuated backscatter profiles, we took the Cloudnet liquid bit algorithm (Illingworth et al., 2007) as a starting point, updated the liquid cloud detection, especially during precipitation events, and developed additional algorithms for discriminating fog, precipitation, and ice. The new algorithms are widely applicable for both operational use and research, e.g. in-cloud icing detection for the wind energy industry and for aviation. The updated algorithm will also be implemented operationally throughout the ACTRIS-Cloudnet network.

Over Helsinki, both observed and forecast cloud cover distributions are U-shaped, indicating that most of the time the sky is either clear or overcast. Overcast conditions are most common in winter, whereas clear (and broken cloud) conditions are more common in summer. Cloud cover is better forecast in winter; however, this is when the solar resource is lower. The measured GHI is strongly influenced by the an- 


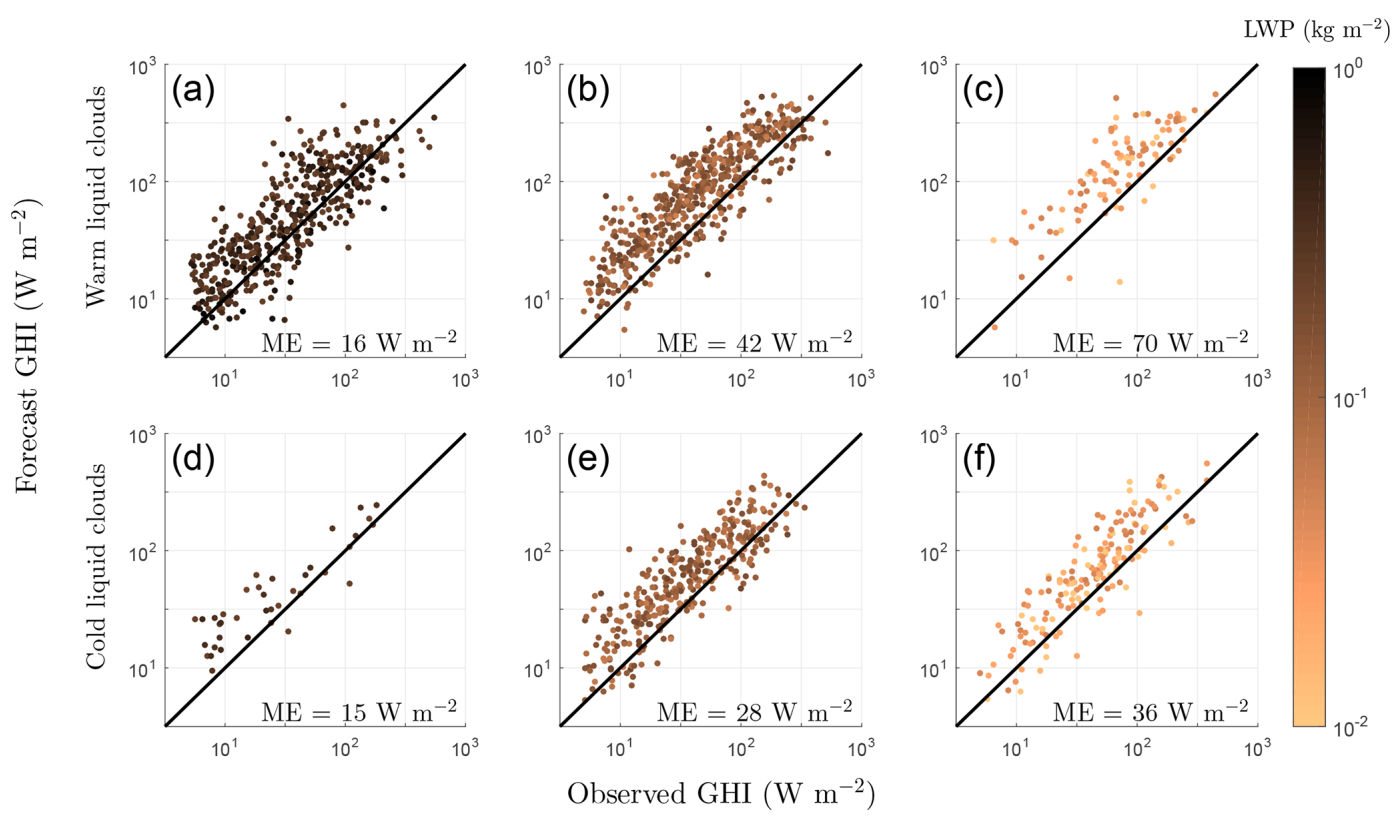

Figure 13. Solar radiation forecast ME versus forecast LWP for different LWP and temperature classes: warm clouds (a)-(c) with cloud base temperature above $0^{\circ} \mathrm{C}$ and cold (supercooled) clouds (d)-(f) with cloud base temperature less than $0{ }^{\circ} \mathrm{C} ; \mathrm{LWP}>0.2 \mathrm{~kg} \mathrm{~m}{ }^{-2}$ (a), (d); $0.2 \mathrm{~kg} \mathrm{~m}^{-2} \geq \mathrm{LWP} \geq 0.05 \mathrm{~kg} \mathrm{~m}^{-2}(\mathbf{b}),(\mathbf{e}) ; \mathrm{LWP}<0.05 \mathrm{~kg} \mathrm{~m}^{-2}$ (c), (f). Colour scale indicates LWP values.

nual solar resource characterized by the northern latitude and annual variations in cloudiness; the absolute solar radiation forecast error tracks GHI, but the relative error is more or less constant throughout the year.

As expected, the bias in forecast GHI is negative when the model overestimates cloud cover (incoming solar radiation is underestimated by the model) and positive when the model underestimates cloud cover. Temporal averaging of the data improves the cloud cover forecasts and decreases the solar radiation forecast errors, as was shown by Hogan et al. (2009). The mean overall bias in the GHI forecast is positive $\left(8 \mathrm{~W} \mathrm{~m}^{-2}\right)$. However, there is a negative bias in forecast GHI for correctly forecast clear cases and a positive bias in correctly forecast overcast cases. A mean overall positive bias would be expected if, on average, the forecast cloud cover was being underestimated, but the forecast cloud cover is usually overestimated on average. This is because the positive GHI bias for the very frequent overcast situations dominates the overall bias. This positive bias occurs for cases in which the model cloud has low values of LWP, and we attribute this bias to the model having LWP values that are too low or the model optical properties for clouds with low LWP being incorrect.

In the future, these methods and analysis can be extended to hundreds of sites across Europe which are now producing ceilometer attenuated backscatter profiles. This analysis will also be performed at Cloudnet stations, which have the advantage in that they have observations of LWP, together with full cloud profiling, enabling the source of the positive bias in clouds with low LWP to be investigated further.
Data availability. The data used in this paper are available on request from FMI (ewan.oconnor@fmi.fi). 
Table A1. Contingency table for skill score calculation. Total number of counts, $n=a+b+c+d$, where $a, b, c$, and $d$ are the number of counts for each situation.

\begin{tabular}{lll}
\hline & $\begin{array}{l}\text { Observed cloud } \\
\text { cover }>0.05\end{array}$ & $\begin{array}{l}\text { Observed cloud } \\
\text { cover } \leq 0.05\end{array}$ \\
\hline $\begin{array}{l}\text { Forecast cloud } \\
\text { cover }>0.05\end{array}$ & $a=$ Hit & $b=$ False alarm \\
\hline $\begin{array}{l}\text { Forecast cloud } \\
\text { cover } \leq 0.05\end{array}$ & $c=$ Miss & $d=$ Correct negative \\
\hline
\end{tabular}

\section{Appendix A: Skill scores and error metrics}

\section{A1 Skill score calculation}

The skill scores in this study are calculated using the generalized skill score equation (Hogan et al., 2009), which for MAESS and MSESS can be simplified to

$S=1-\frac{x}{x_{\mathrm{r}}}$

where $x=(\mathrm{frc}-\mathrm{obs})^{2}$ for MSESS and $x=|\mathrm{frc}-\mathrm{obs}|$ for MAESS, and the values for the random forecast, $x_{\mathrm{r}}$, are calculated from elements of the contingency table $x_{\mathrm{r}}=\frac{a+b}{n}$. $\frac{a+c}{n}+\frac{d+c}{n} \cdot \frac{d+b}{n}$ for both MSESS and MAESS. The values obs and frc refer to the observed and forecast values of the variable of interest, e.g. cloud cover.

\section{A2 Error metrics}

$\mathrm{MAE}=\frac{1}{n} \sum_{t=1}^{n}|\mathrm{frc}-\mathrm{obs}|$

MAPE $=\frac{1}{n} \sum_{t=1}^{n} \frac{\mathrm{frc}-\mathrm{obs}}{\mathrm{obs}} \cdot 100$,

$\mathrm{ME}=\frac{1}{n} \sum_{t=1}^{n}(\mathrm{frc}-\mathrm{obs})$,

RMSE $=\sqrt{\frac{1}{n} \sum_{t=1}^{n}(\mathrm{frc}-\mathrm{obs})^{2}}$. 
Author contributions. All authors conceived and designed the analysis. MT and EOC obtained the data, and MT performed the analysis. MT wrote the paper with input from all authors. EOC and VS conceived the study and were in charge of overall direction and planning.

Competing interests. The authors declare that they have no conflict of interest.

Acknowledgements. This work was supported by the Maj and Tor Nessling Foundation (grant 201700032) and by the European Commission via project ACTRIS2 (grant agreement no. 654109). Victoria A. Sinclair is funded by the Academy of Finland (project no. 307331). We acknowledge ECMWF for providing model output from IFS and FMI for providing ceilometer and solar radiation observations.

Edited by: Jui-Yuan Christine Chiu

Reviewed by: three anonymous referees

\section{References}

Ahlgrimm, M. and Forbes, R.: The impact of low clouds on surface shortwave radiation in the ECMWF model, Mon. Weather Rev., 140, 3783-3794, https://doi.org/10.1175/MWR-D-11-00316.1, 2012.

Barrett, A. I., Hogan, R. J., and Forbes, R. M.: Why are mixed-phase altocumulus clouds poorly predicted by large-scale models? Part 1. Physical processes, J. Geophys. Res.-Atmos., 122, 9903-9926, https://doi.org/10.1002/2016JD026321, 2017.

Boilley, A. and Wald, L.: Comparison between meteorological reanalyses from ERA-Interim and MERRA and measurements of daily solar irradiation at surface, Renew. Energ., 75, 135-143, https://doi.org/10.1016/j.renene.2014.09.042, 2015.

Bozzo, A., Remy, S., Benedetti, A., Flemming, J., Bechtold, P., Rodwell, M., and Morcrette, J.-J.: Implementation of a CAMSbased aerosol climatology in the IFS, ECMWF, Technical Memorandum, 801, https://doi.org/10.21957/84ya94mls, 2017.

Forbes, R. M. and Ahlgrimm, M.: On the representation of high-latitude boundary layer mixed-phase cloud in the ECMWF global model, Mon. Weather Rev., 142, 3425-3445, https://doi.org/10.1175/MWR-D-13-00325.1, 2014.

Frank, C. W., Wahl, S., Keller, J. D., Pospichal, B., Hense, A., and Crewell, S.: Bias correction of a novel European reanalysis data set for solar energy applications, Sol. Energy, 164, 12-24, https://doi.org/10.1016/j.solener.2018.02.012, 2018.

Hogan, R. and Bozzo, A.: ECRAD: A new radiation scheme for the IFS, ECMWF, Technical Memorandum, 787, https://doi.org/10.21957/whntqkfdz, 2016.

Hogan, R. J. and Illingworth, A. J.: Deriving cloud overlap statistics from radar, Q. J. Roy. Meteorol. Soc., 126, 2903-2909, https://doi.org/10.1002/qj.49712656914, 2000.

Hogan, R. J., Illingworth, A. J., O'connor, E. J., and Baptista, J. P. V. P.: Characteristics of mixed-phase clouds. II: A climatology from ground-based lidar, Q. J. Roy. Meteorol. Soc., 129, 21172134, https://doi.org/10.1256/qj.01.209, 2003.
Hogan, R. J., O'Connor, E. J., and Illingworth, A. J.: Verification of cloud-fraction forecasts, Q. J. Roy. Meteorol. Soc., 135, 14941511, https://doi.org/10.1002/qj.481, 2009.

Illingworth, A. J., Hogan, R. J., O’Connor, E., Bouniol, D., Brooks, M. E., Delanoé, J., Donovan, D. P., Eastment, J. D., Gaussiat, N., Goddard, J. W. F., Haeffelin, M., Baltink, H. K., Krasnov, O. A., Pelon, J., Piriou, J.-M., Protat, A., Russchenberg, H. W. J., Seifert, A., Tompkins, A. M., van Zadelhoff, G.-J., Vinit, F., Willén, U., Wilson, D. R., and Wrench, C. L.: Cloudnet, B. Am. Meteor. Soc., 88, 883-898, https://doi.org/10.1175/BAMS-88-6883, 2007.

Illingworth, A. J., Cimini, D., Gaffard, C., Haeffelin, M., Lehmann, V., Löhnert, U., O'Connor, E. J., and Ruffieux, D.: Exploiting existing ground-based remote sensing networks to improve highresolution weather forecasts, B. Am. Meteor. Soc., 96, 21072125, https://doi.org/10.1175/BAMS-D-13-00283.1, 2015.

Illingworth, A. J., Cimini, D., Haefele, A., Haeffelin, M., Hervo, M., Kotthaus, S., Löhnert, U., Martinet, P., Mattis, I., O'Connor, E. J., and Potthast, R.: How can existing ground-based profiling instruments improve European weather forecasts?, B. Am. Meteor. Soc., https://doi.org/10.1175/BAMS-D-17-0231.1, 2019.

Jia, B., Xie, Z., Dai, A., Shi, C., and Chen, F.: Evaluation of satellite and reanalysis products of downward surface solar radiation over East Asia: Spatial and seasonal variations, J. Geophys. Res.Atmos., 118, 3431-3446, https://doi.org/10.1002/jgrd.50353, 2013.

Kleissl, J.: Solar energy forecasting and resource assessment, Academic Press, 1-462, 2013.

Kotthaus, S. and Grimmond, C. S. B.: Atmospheric boundarylayer characteristics from ceilometer measurements. Part 1: A new method to track mixed layer height and classify clouds, Q. J. Roy. Meteorol. Soc., 144, 1525-1538, https://doi.org/10.1002/qj.3299, 2018.

Kotthaus, S., O’Connor, E., Münkel, C., Charlton-Perez, C., Haeffelin, M., Gabey, A. M., and Grimmond, C. S. B.: Recommendations for processing atmospheric attenuated backscatter profiles from Vaisala CL31 ceilometers, Atmos. Meas. Tech., 9, 37693791, https://doi.org/10.5194/amt-9-3769-2016, 2016.

Long, C. N. and Shi, Y.: An automated quality assessment and control algorithm for surface radiation measurements, Open Atmos. Sci. J., 2, 23-37, https://doi.org/10.2174/1874282300802010023, 2008.

López, G. and Batlles, F. J.: Estimating solar radiation from MODIS data, Energy Proced., 49, 2362-2369, https://doi.org/10.1016/j.egypro.2014.03.250, 2014.

Martucci, G., Milroy, C., and O'Dowd, C. D.: Detection of cloud-base height using jenoptik CHM15K and Vaisala CL31 ceilometers, J. Atmos. Ocean. Tech., 27, 305-318, https://doi.org/10.1175/2009JTECHA1326.1, 2010.

Mather, J. H. and Voyles, J. W.: The Arm climate research facility: A review of structure and capabilities, B. Am. Meteor. Soc., 94, 377-392, https://doi.org/10.1175/BAMS-D-11-00218.1, 2013.

Mittermaier, M.: A critical assessment of surface cloud observations and their use for verifying cloud forecasts, Q. J. Roy. Meteorol. Soc., 138, 1794-1807, https://doi.org/10.1002/qj.1918, 2012.

Morcrette, C. J., O'Connor, E. J., and Petch, J. C.: Evaluation of two cloud parametrization schemes using ARM and CloudNet observations, Q. J. Roy. Meteorol. Soc., 138, 964-979, https://doi.org/10.1002/qj.969, 2014. 
Morcrette, J.-J., Barker, H. W., Cole, J. N. S., Iacono, M. J., and Pincus, R.: Impact of a new radiation package, McRad, in the ECMWF Integrated Forecasting System, Mon. Weather Rev., 136, 4773-4798, https://doi.org/10.1175/2008MWR2363.1, 2008.

Morille, Y., Haeffelin, M., Drobinski, P., and Pelon, J.: STRAT: An automated algorithm to retrieve the vertical structure of the atmosphere from single-channel lidar data, J. Atmos. Ocean. Tech., 24, 761-775, https://doi.org/10.1175/JTECH2008.1, 2007.

Münkel, C., Eresmaa, N., Räsänen, J., and Karppinen, A.: Retrieval of mixing height and dust concentration with lidar ceilometer, Bound.-Lay. Meteorol., 124, 117-128, https://doi.org/10.1007/s10546-006-9103-3, 2007.

Müller, R., Pfeifroth, U., Träger-Chatterjee, C., Trentmann, J., and Cremer, R.: Digging the METEOSAT treasure - 3 decades of solar surface radiation, Remote Sens.-Basel, 7, 8067-8101, https://doi.org/10.3390/rs70608067, 2015.

Murphy, A. H.: Skill scores based on the mean square error and their relationships to the correlation coefficient, Mon. Weather Rev., 116, 2417-2424, https://doi.org/10.1175/15200493(1988)116<2417:SSBOTM>2.0.CO;2, 1988.

O'Connor, E. J., Illingworth, A. J., and Hogan, R. J.: A technique for autocalibration of cloud lidar, J. Atmos. Ocean. Tech., 21, 777-786, https://doi.org/10.1175/15200426(2004)021<0777:ATFAOC>2.0.CO;2, 2004.

Ohmura, A., Dutton, E. G., Forgan, B., Fröhlich, C., Gilgen, H., Hegner, H., Heimo, A., König-Langlo, G., McArthur, B., Müller, G., Philipona, R., Pinker, R., Whitlock, C. H., Dehne, K., and Wild, M.: Baseline Surface Radiation Network (BSRN/WCRP): New precision radiometry for climate research, B. Am. Meteor. Soc., 79, 2115-2136, https://doi.org/10.1175/15200477(1998)079<2115:BSRNBW>2.0.CO;2, 1998.
Posselt, R., Mueller, R., Stöckli, R., and Trentmann, J.: Remote sensing of solar surface radiation for climate monitoring - the CM-SAF retrieval in international comparison, Remote Sens. Environ., 118, 186-198, https://doi.org/10.1016/j.rse.2011.11.016, 2012.

Rontu, L. and Lindfors, A. V.: Comparison of radiation parametrizations within the HARMONIE-AROME NWP model, Adv. Sci. Res., 15, 81-90, https://doi.org/10.5194/asr-15-81-2018, 2018.

Urraca, R., Huld, T., Gracia-Amillo, A., de Pison, F. J. M., Kaspar, F., and Sanz-Garcia, A.: Evaluation of global horizontal irradiance estimates from ERA5 and COSMO-REA6 reanalyses using ground and satellite-based data, Sol. Energy, 164, 339-354, https://doi.org/10.1016/j.solener.2018.02.059, 2018.

Van Tricht, K., Gorodetskaya, I. V., Lhermitte, S., Turner, D. D., Schween, J. H., and Van Lipzig, N. P. M.: An improved algorithm for polar cloud-base detection by ceilometer over the ice sheets, Atmos. Meas. Tech., 7, 1153-1167, https://doi.org/10.5194/amt7-1153-2014, 2014.

Van Weverberg, K., Morcrette, C. J., Petch, J., Klein, S. A., Ma, H.-Y., Zhang, C., Xie, S., Tang, Q., Gustafson, W. I., Qian, Y., Berg, L. K., Liu, Y., Huang, M., Ahlgrimm, M., Forbes, R., Bazile, E., Roehrig, R., Cole, J., Merryfield, W., Lee, W.-S., Cheruy, F., Mellul, L., Wang, Y.-C., Johnson, K., and Thieman, M. M.: CAUSES: Attribution of surface radiation biases in NWP and climate models near the U.S. Southern Great Plains, J. Geophys. Res.-Atmos., 123, 3612-3644, https://doi.org/10.1002/2017JD027188, 2018. 